\title{
SOME CHARACTERIZATIONS OF PARTIAL EXCHANGEABILITY
}

\author{
B. G. IVANOFF and N. C. WEBER
}

(Received 14 June 1994; revised 21 April 1995)

Communicated by R. A. Maller

\begin{abstract}
Kallenberg [2] introduced the concept of $\mathscr{F}$-exchangeable sequences of random variables and produced some characterizations of $\mathscr{F}$-exchangeability in terms of stopping times. In this paper ways of extending the concept of $\mathscr{F}$-exchangeability to doubly indexed arrays of random variables are explored and some characterizations obtained for row and column exchangeable arrays, weakly exchangeable arrays and separately exchangeable continuous processes.
\end{abstract}

1991 Mathematics subject classification (Amer. Math. Soc.): 60 G09.

Keywords and phrases: Row and column exchangeable arrays, stopping times, weakly exchangeable arrays, martingales, separately exchangeable processes.

\section{Introduction}

A sequence of random variables $X=\left(X_{1}, X_{2} \ldots\right)$ is said to be exchangeable if ( $X_{1}$, $\left.X_{2}, \ldots\right) \stackrel{?}{=}\left(X_{\pi(1)}, X_{\pi(2)}, \ldots\right)$ for any permutation $\pi$ of the positive integers that leaves all but a finite number of integers fixed. A finite sequence $\left(X_{1}, X_{2}, \ldots, X_{n}\right)$ is finitely exchangeable if $\left(X_{1}, X_{2}, \ldots, X_{n}\right) \stackrel{\mathscr{Q}}{=}\left(X_{\pi(1)}, \ldots, X_{\pi(n)}\right)$, where $\pi$ is any permutation of $(1,2, \ldots, n)$. Any finite subsequence of an infinite exchangeable sequence is finitely exchangeable but finitely exchangeable sequences cannot necessarily be embedded into an infinite sequence of exchangeable random variables.

Kingman [3] gave a detailed account of infinite and finite exchangeability along with a discussion of various characterizations that have been developed. Kallenberg [2] introduced the concept of $\mathscr{F}$-exchangeability for sequences adapted to some filtration $\mathscr{F}=\left(\mathscr{F}_{0}, \mathscr{F}_{1}, \ldots\right): X$ is said to be $\mathscr{F}$-exchangeable if $\left(X_{n+1}, X_{n+2}, \ldots\right)$ is conditionally exchangeable given $\mathscr{F}_{n}$. Kallenberg noted that all $\mathscr{F}$-exchangeable se-

(C) 1996 Australian Mathematical Society 0263-6115/96 \$A2.00+0.00 
quences were exchangeable and if $\theta_{n}$ denotes the shift operator, that is, $\theta_{n} \circ X=\left(X_{n+1}\right.$, $\left.X_{n+2}, \ldots\right)$, then the infinite sequence $X$ is $\mathscr{F}$-exchangeable if and only if $\theta_{\tau} \circ X \stackrel{\mathscr{D}}{=} X$ for every $\mathscr{F}$-stopping time $\tau$. This characterization was shown to be an extension of a characterization of Dacunha-Castelle [1] for infinite exchangeable sequences. Further, Kallenberg [2] gave some characterizations for finitely $\mathscr{F}$-exchangeable sequences in terms of stopping times and reflections.

In this note we consider ways of extending the concept of $\mathscr{F}$-exchangeability to partially exchangeable arrays; that is, row and column exchangeable (RCE) arrays and weakly exchangeable arrays. This is an interesting problem, for in the doubly indexed situation we find different definitions of $\mathscr{F}$-exchangeability are needed to deal with the finite and infinite cases. Moreover, there does not appear to be a generalization of Kallenberg's characterization for infinite weakly exchangeable arrays. In Section 2 we consider the infinite case and provide a characterization analogous to Theorem 2.1 in Kallenberg [2] for RCE arrays. In Section 3 we consider the case of finite arrays and extend Kallenberg's Proposition 2.2 to obtain characterizations for RCE and weakly exchangeable arrays, and in Section 4 we apply these characterizations to obtain results for continuous processes.

\section{Infinite arrays}

An array $X=\left\{X_{i j} ; i \geq 1, j \geq 1\right\}$ of random elements taking values in an arbitrary measurable space $(G, \mathscr{S})$ is row and column exchangeable, RCE, if $X \stackrel{\mathscr{Q}}{=}\left\{X_{\pi(i), \sigma(j)}\right\}$ where $\pi$ and $\sigma$ are separate permutations of the positive integers which leave all but a finite number of the integers fixed. An array $X$ is said to be weakly exchangeable if $X \stackrel{\mathscr{D}}{=}\left\{X_{\pi(i), \pi(j)}\right\}$ where $\pi$ is as above. Thus all infinite RCE arrays are weakly exchangeable.

Following Kallenberg [2] we assume $X$ is adapted to a filtration $\mathscr{F}=\left\{\mathscr{F}_{i j} ; i \geq\right.$ $0, j \geq 0\}$. Let $\theta_{m n}$ denote the shift operator: $\theta_{m n} \circ X=\left\{X_{m+i, n+j} ; i \geq 1, j \geq 1\right\}$. We say $X$ is weak $\mathscr{F}$-RCE if $\theta_{m n} \circ X$ is conditionally RCE given $\mathscr{F}_{m n}$. If $X$ is weak $\mathscr{F}$-RCE then $X$ is also unconditionally a row and column exchangeable array. Furthermore, we say $X$ is weak $\mathscr{F}$-weakly exchangeable if $\theta_{m n} \circ X$ is conditionally weakly exchangeable given $\mathscr{F}_{m n}$. Our first result is a characterization of weak $\mathscr{F}$ RCE arrays in terms of random shifts and so can be thought of as an extension of Theorem 2.1 of Kallenberg [2] to this partial exchangeability situation. The random vector $(S, T)$ taking values in $Z_{+}^{2}$ is an adapted random time with respect to $\mathscr{F}$ if $\{S=i, T=j\} \in \mathscr{F}_{i j}, i, j \geq 1$.

Before stating our main theorem we give the following result on (2-parameter) martingales. 
DEFINITION. An integrable process $X=\left\{X_{i j}: i, j \geq 0\right\}$ adapted to a filtration $\mathscr{F}$ is an $(\mathscr{F}-)$ martingale if for all $i, j, k, \ell$ such that $i \leq k, j \leq \ell, E\left(X_{k \ell} \mid \mathscr{F}_{i j}\right)=X_{i j}$ a.s.

LemMA 1. $M$ is a martingale if $E M_{S, T}=E M_{0,0}$, for all adapted, bounded, random times $(S, T)$.

PROOF. Let $m \leq m^{\prime}$ and $n \leq n^{\prime}$ be given and let $A \in \mathscr{F}_{m n}$. Set $(S, T)=$ $(m, n) I(A)+\left(m^{\prime}, n^{\prime}\right) I\left(A^{c}\right) \cdot(S, T)$ is an adapted random time with respect to $\mathscr{F}$. Now

$$
E M_{0,0}=E M_{m^{\prime} n^{\prime}}=E M_{S, T}=E M_{m n} I(A)+E M_{m^{\prime} n^{\prime}} I\left(A^{c}\right),
$$$$
\text { so } \quad E M_{m^{\prime} n^{\prime}} I(A)=E M_{m n} I(A) \text {. }
$$

Thus $E\left(M_{m^{\prime} n^{\prime}} \mid \mathscr{F}_{m n}\right)=M_{m n}$ a.s., since (1) holds for arbitrary $A \in \mathscr{F}_{m n}$, and so $M$ is a martingale.

THEOREM 1. $X$ is a weak $\mathscr{F}$-RCE array if and only if $\theta_{S, T} \circ X \stackrel{\mathscr{D}}{=} X$ for all adapted, bounded, random times $(S, T)$.

PROOF. First assume that $X$ is weak $\mathscr{F}$-RCE and let $(S, T)$ be a bounded, adapted random time, $S, T \leq m$ a.s. Let $f: G^{\infty} \times G^{\infty} \rightarrow R$ be a bounded function of the first $n$ coordinates in each direction, $n \in N$. Then

$$
\begin{aligned}
E\left(f\left(\theta_{S, T} \circ X\right)\right) & =\sum_{i=1}^{m} \sum_{j=1}^{m} E\left[f\left(\theta_{S, T} \circ X\right) ; S=i, T=j\right] \\
& =\sum_{i=1}^{m} \sum_{j=1}^{m} E\left[f\left(\theta_{i, j} \circ X\right) ; S=i, T=j\right] \\
& =\sum_{i=1}^{m} \sum_{j=1}^{m} E\left[f\left(\theta_{m, m} \circ X\right) ; S=i, T=j\right] \\
& =E f\left(\theta_{m, m} \circ X\right) \\
& =E f(X),
\end{aligned}
$$

where the third equality follows since given $\mathscr{F}_{i j}, \theta_{i j} \circ X$ is RCE. By a monotone class argument, this extends to arbitrary bounded $f$.

Conversely, assume $\theta_{S, T} \circ X \stackrel{\mathscr{Q}}{=} X$ for arbitrary, finite, adapted, bounded random times $(S, T)$. For fixed bounded $f: G^{\infty} \times G^{\infty} \rightarrow R$, define

$$
M_{m n}=E\left[f\left(\theta_{m, n} \circ X\right) \mid \mathscr{F}_{m, n}\right], \quad m, n \in N .
$$


For any adapted, bounded, random time $(S, T)$,

$$
\begin{aligned}
E M_{S, T} & =\sum_{m} \sum_{n} E\left[M_{m n} ; S=m, T=n\right] \\
& =\sum_{m} \sum_{n} E\left[E\left(f\left(\theta_{m n} \circ X\right) ; S=m, T=n \mid \mathscr{F}_{m n}\right)\right] \\
& =E f\left(\theta_{S, T} \circ X\right) \\
& =E f(X), \quad \text { by assumption. }
\end{aligned}
$$

Thus from Lemma $1, M$ is a martingale and so for any $k, \ell \geq 0$,

$$
\begin{aligned}
E\left[f\left(\theta_{m+k, n+\ell} \circ X\right) \mid \mathscr{F}_{m n}\right] & =E\left[E\left(f\left(\theta_{m+k, n+\ell} \circ X\right) \mid \mathscr{F}_{m+k, n+\ell}\right) \mid \mathscr{F}_{m n}\right] \\
& =E\left[M_{m+k, n+\ell} \mid \mathscr{F}_{m n}\right] \\
& =M_{m n}, \quad \text { for all } k, \ell \in N .
\end{aligned}
$$

To complete the proof, we will show that conditional on $\mathscr{F}_{m n}$ the columns of $\theta_{m, n} \circ X$ are exchangeable, as a similar argument can be used to establish that, given $\mathscr{F}_{m n}$, the rows of $\theta_{m, n} \circ X$ are exchangeable.

For fixed $n$, the sequence $f\left(\theta_{k, n} \circ X\right), k \geq 1$ is stationary and bounded, so by the pointwise ergodic theorem

$$
\lim _{u \rightarrow \infty} \frac{1}{u} \sum_{k=1}^{u} f\left(\theta_{m+k, n} \circ X\right)=E\left[f\left(X_{n}^{(1)}\right) \mid \mathscr{C}_{n}\right] \text { a.s., }
$$

where $X_{n}^{(1)}=\left\{X_{i j} ; i \geq 1, j>n\right\}$ and $\mathscr{C}_{n}$ denotes the $\sigma$-field of $X_{n}^{(1)}$-measurable sets that are invariant under column shifts. From (3), and the dominated convergence theorem,

$$
E\left[E\left[f\left(X_{n}^{(1)}\right) \mid \mathscr{C}_{n}\right] \mid \mathscr{F}_{m n}\right]=M_{m n}=E\left[f\left(\theta_{m n} \circ X\right) \mid \mathscr{F}_{m n}\right]
$$

If $g$ is a bounded function which is shift invariant under column shifts, then

$$
\begin{aligned}
E\left[f\left(\theta_{m, n} \circ \bar{X}\right) g\left(X_{n}^{(1)}\right) \mid \mathscr{F}_{m n}\right] & =E\left[f\left(\theta_{m, n} \circ X\right) g\left(\theta_{m, n} \circ X\right) \mid \mathscr{F}_{m n}\right] \\
& =E\left[E\left(f\left(X_{n}^{(1)}\right) g\left(X_{n}^{(1)}\right) \mid \mathscr{C}_{n}\right) \mid \mathscr{F}_{m n}\right], \\
& =E\left[E\left(f\left(X_{n}^{(1)}\right) \mid \mathscr{C}_{n}\right) g\left(X_{n}^{(1)}\right) \mid \mathscr{F}_{m n}\right],
\end{aligned}
$$

which means that

$$
E\left[f\left(\theta_{m, n} \circ X\right) \mid \mathscr{F}_{m n} \vee \mathscr{C}_{n}\right]=E\left(f\left(X_{n}^{(1)}\right) \mid \mathscr{C}_{n}\right) \text { a.s. } .
$$


Write $X_{n}^{(1)}=\left[\mathbf{X}_{1 n}, \mathbf{X}_{2 n}, \mathbf{X}_{3 n}, \ldots\right]$. Consider arbitrary $u \in N$ and $B_{1}, \ldots, B_{u} \in \mathscr{S}^{\infty}$ :

(6)

$$
\begin{aligned}
P\left[\bigcap_{k=1}^{u}\left\{\mathbf{X}_{m+k, n} \in B_{k}\right\} \mid \mathscr{F}_{m n} \vee \mathscr{C}_{n}\right] \\
\quad=E\left[E\left[\prod_{k=1}^{u} I\left(\mathbf{X}_{m+k, n} \in B_{k}\right) \mid \mathscr{F}_{m+u-1, n} \vee \mathscr{C}_{n}\right] \mid \mathscr{F}_{m, n} \vee \mathscr{C}_{n}\right] \\
=E\left[\prod_{k=1}^{u-1} I\left(\mathbf{X}_{m+k, n} \in B_{k}\right) \cdot E\left(I\left(\mathbf{X}_{1 n} \in B_{u}\right) \mid \mathscr{C}_{n}\right) \mid \mathscr{F}_{m, n} \vee \mathscr{C}_{n}\right], \\
\quad \text { from }(5) \text { with } f(X)=I\left(\mathbf{X}_{10} \in B_{u}\right), \\
\quad=E\left[\prod_{k=1}^{u-1} I\left(\mathbf{X}_{m+k, n} \in B_{k}\right) \mid \mathscr{F}_{m, n} \vee \mathscr{C}_{n}\right] \cdot P\left(\mathbf{X}_{1 n} \in B_{u} \mid \mathscr{C}_{n}\right) \\
=\prod_{k=1}^{u} P\left(\mathbf{X}_{k n} \in B_{k} \mid \mathscr{C}_{n}\right),
\end{aligned}
$$

since $P\left(\mathbf{X}_{1 n} \in B_{k} \mid \mathscr{C}_{n}\right)=P\left(\mathbf{X}_{k n} \in B_{k} \mid \mathscr{C}_{n}\right)$, by shift invariance.

Now as in Kallenberg's proof for fixed $h \in N$ and $m>0$, let $\mathscr{D} \subset \mathscr{S}^{\infty} \times \cdots \times \mathscr{S}^{\infty}$, ( $h$ terms), be the class of sets $A$ such that

$$
\begin{aligned}
& P\left(\left(\mathbf{X}_{m+1, n}, \mathbf{X}_{m+2, n}, \ldots, \mathbf{X}_{m+h, n}\right) \in A \mid \mathscr{F}_{m n}\right) \\
& \quad=P\left(\left(\mathbf{X}_{m+\pi(1), n}, \ldots, \mathbf{X}_{m+\pi(h), n}\right) \in A \mid \mathscr{F}_{m n}\right) \text { a.s. }
\end{aligned}
$$

for all $m, n$ and every permutation $\pi$ of the indices $1, \ldots, h$. Equation (6) implies that every product set belongs to $\mathscr{D}$ and so it follows that $\mathscr{D}=\mathscr{S}^{\infty} \times \cdots \times \mathscr{S}^{\infty}$. Since $h$ and $m$ were arbitrary it follows that the columns of $\theta_{m, n} \circ X$ are exchangeable, given $\mathscr{F}_{m n}$.

In the above proof we only need $\theta_{S, T} \circ X \stackrel{\mathscr{D}}{=} X$ for adapted random times taking two distinct ordered values. Thus if $A \in \mathscr{F}_{m n}$ and $(S, T)=(m, n) I(A)+\left(m^{\prime}, n^{\prime}\right) I\left(A^{c}\right)$, where $m^{\prime} \geq m, n^{\prime} \geq n$ we get the equivalent condition

$$
E\left[f\left(\theta_{m, n} \circ X\right) I(A)\right]=E\left[f\left(\theta_{m^{\prime}, n^{\prime}} \circ X\right) I(A)\right]
$$

for arbitrary $A \in \mathscr{F}_{m n}$ and bounded $f: G^{\infty} \times G^{\infty} \rightarrow R$. Thus $X$ is weak $\mathscr{F}$-RCE if and only if given $\mathscr{F}_{m n}, \theta_{m, n} \circ X \stackrel{\mathscr{D}}{=} \theta_{u, v} \circ X$, for all $(m, n),(u, v)$ such that $m \leq u, n \leq v$.

If $X$ is RCE then the columns form an exchangeable sequence, as do the rows. Is it possible to characterize an RCE array via row and column conditions separately? A 
partial response to this question is given in the following lemma. Let $\mathscr{F}_{i}^{1}=\bigvee_{j} \mathscr{F}_{i j}$ and $\mathscr{F}_{j}^{2}=\bigvee_{i} \mathscr{F}_{i j} i, j \geq 0$ and assume $\mathscr{F}$ satisfies the condition

(F4) : $\mathscr{F}_{i}^{1}$ is conditionally independent of $\mathscr{F}_{j}^{2}$ given $\mathscr{F}_{i j}$ for all $i, j$.

This condition is equivalent to the property of commutation of the conditional expectation operators. Note that if $(S, T)$ is an adapted random time then $S$ is an $\mathscr{F}^{1}$ stopping time and $T$ is an $\mathscr{F}^{2}$ stopping time.

LEMMA 2. If $\mathscr{F}$ satisfies the condition (F4), and $X$ is $\mathscr{F}^{1}$-exchangeable as a column process and $\mathscr{F}^{2}$-exchangeable as a row process, then $X$ is a weak $\mathscr{F}-\mathrm{RCE}$ array.

PrOOF. For permutations $\pi$ and $\sigma$, and any measurable set $A$,

$$
\begin{array}{rlrl}
P\left(\left(X_{m+\pi(i), n+\sigma(j)}\right) \in A \mid \mathscr{F}_{m n}\right) & & \\
& =E\left(P\left(\left(X_{m+\pi(i), n+\sigma(j)}\right) \in A \mid \mathscr{F}_{m}^{1}\right) \mid \mathscr{F}_{n}^{2}\right), & & \text { by }(\mathrm{F} 4) \\
& =E\left(P\left(\left(X_{m+i, n+\sigma(j)}\right) \in A \mid \mathscr{F}_{m}^{1}\right) \mid \mathscr{F}_{n}^{2}\right), & & \text { by } \mathscr{F}^{1} \text {-exchangeability } \\
& =E\left(P\left(\left(X_{m+i, n+\sigma(j)}\right) \in A \mid \mathscr{F}_{n}^{2}\right) \mid \mathscr{F}_{m}^{1}\right) & & \\
& =E\left(P\left(\left(X_{m+i, n+j}\right) \in A \mid \mathscr{F}_{n}^{2}\right) \mid \mathscr{F}_{m}^{1}\right), & & \text { by } \mathscr{F}^{2} \text {-exchangeability } \\
& =P\left(\left(X_{m+i, n+j}\right) \in A \mid \mathscr{F}_{m n}\right), & & \text { by (F4) }
\end{array}
$$

so $X$ is weak $\mathscr{F}$-RCE.

The converse to Lemma 2 is not true, even under (F4). The following example is an array which is weak $\mathscr{F}$-RCE but not $\mathscr{F}^{1}$-exchangeable.

EXAMPLE. Let $\left\{\xi_{i}\right\},\left\{\eta_{i}\right\}$ and $\left\{\lambda_{i j}\right\}$ be independent $U(0,1)$ random variables. Let $X_{i j}=\xi_{i} \eta_{j} \lambda_{i j}$ and $\mathscr{F}_{i j}=\sigma\left(\xi_{k}, k \leq i ; \eta_{\ell}, \lambda_{h \ell}, \ell \leq j, h \geq 1\right)$ for $i, j \geq 1$. Given $\mathscr{F}_{i j}$, $\theta_{i j} \circ X$ is RCE. Now $\mathscr{F}_{i}^{1}=\sigma\left(\xi_{k}, k \leq i ; \eta_{\ell}, \lambda_{h \ell}, h, \ell \geq 1\right), i \geq 1$. Also

$$
\mathbf{X}_{n}^{(1)}=\left(\begin{array}{c}
\vdots \\
X_{n 2} \\
X_{n 1}
\end{array}\right)=\xi_{n}\left(\begin{array}{c}
\vdots \\
\eta_{2} \lambda_{n 2} \\
\eta_{1} \lambda_{n 1}
\end{array}\right)
$$

and so given $\mathscr{F}_{n-1}^{1}$, the columns $\mathbf{X}_{n}^{(1)}, \mathbf{X}_{n+1}^{(1)}, \ldots$ are not identically distributed and so are not exchangeable. However $\mathscr{F}_{j}^{2}=\sigma\left(\xi_{k}, \eta_{\ell}, \lambda_{h \ell}, \ell \leq j, h, k \geq 1\right)$ and so $\mathscr{F}$ satisfies (F4).

Is there a characterization of weak $\mathscr{F}$-weak exchangeability in terms of random shifts? To maintain symmetry we need any such shifts to be along the diagonal. If $T$ is a stopping time with respect to $\left(\mathscr{F}_{i i}\right)$, then a necessary condition for weak $\mathscr{F}$-weak 
exchangeability is $\theta_{T, T} \circ X \stackrel{\mathscr{D}}{=} X$; but this condition is not sufficient. To see this, consider $X=\left(X_{i j}\right)$, defined by

$$
X_{i j}= \begin{cases}\xi_{i}, & j \geq i, \\ \eta_{j}, & j<i,\end{cases}
$$

where $\xi_{1}, \xi_{2}, \ldots, \eta_{1}, \eta_{2}, \ldots$ are independent $U(0,1)$ random variables. If $\mathscr{F}$ is the induced filtration, then $\theta_{T, T} \circ X \stackrel{\mathscr{D}}{=} X$; but $X$ is not weakly exchangeable.

\section{Finite arrays}

An array $X=\left\{X_{i j} ; 1 \leq i \leq m, 1 \leq j \leq r\right\}$ of random elements taking values in an arbitrary measurable space $(G, \mathscr{S})$ is finitely RCE if $X \stackrel{\mathscr{D}}{=}\left\{X_{\pi(i), \sigma(j)}\right\}$, where $\pi$ is any permutation of $(1,2, \ldots, m)$ and $\sigma$ is any permutation of $(1,2, \ldots, r)$. If $m=r$, then $X$ is finitely weakly exchangeable if $X \stackrel{\mathscr{D}}{=}\left\{X_{\pi(i), \pi(j)}\right\}$. For finite arrays the shifted array does not even have the same dimensions as the original one, and so characterizations like those developed for infinite arrays are not possible. Following Kallenberg we look for characterizations in the finite case based on reflection properties. The extra complication in the case of finite arrays is that reflecting the upper rows of the array while holding the first $i$ rows fixed, and similarly reflecting the upper columns of the array while holding the first $j$ columns fixed, not only affects the upper quadrant of the array, but also impacts on the shoulder regions; that is, the set of variables in the first $i$ rows that are not in the first $j$, columns or in the first $j$ columns but not in the first $i$ rows. For this reason we introduce a stronger version of $\mathscr{F}$-exchangeability based on shells.

Definitions. 1. A finite array $X$ is $\mathscr{F}$-RCE if for all $i \leq m-1, j \leq r-1$ the conditional distribution of the shell, $\left\{X_{u v} ; i<u \leq m\right.$ or $\left.j<v \leq r\right\}$, given $\mathscr{F}_{i j}$, is invariant under finite permutations of rows that leave the first $j$ rows invariant and/or permutations of the columns that leave the first $i$ columns invariant.

2. A finite square array $X$ is $\mathscr{F}$-weakly exchangeable if for all $i$ the conditional distribution of the shell, $\left\{X_{u v} ; i<u \leq m\right.$ or $\left.i<v \leq m\right\}$, given $\mathscr{F}_{i i}$, is invariant under the same finite permutation of the rows and columns of the shell that leave the first $i$ rows and columns invariant.

3. A finite array is weak $\mathscr{F}$-RCE if for all $i \leq m-1, j \leq r-1$ the array $\left\{X_{u v}: i<u \leq m, j<v \leq r\right\}$ is conditionally RCE given $\mathscr{F}_{i j}$.

4. A finite square array $X$ is weak $\mathscr{F}$-weakly exchangeable if the array $\left\{X_{u v}: i<\right.$ $u \leq m, i<v \leq m\}$ is conditionally weakly exchangeable given $\mathscr{F}_{i i}$. 
Note that in the first two cases, although the first $i$ columns are fixed, the elements within the columns may be permuted. For example, under Definition 1 the elements in the first column in rows $j+1, \ldots, r$ may be permuted by row interchanges and similarly the elements of the first row in columns $i+1, \ldots, m$ may be interchanged by column permutations. Clearly, if $X$ is $\mathscr{F}$-RCE, then $X$ is weak $\mathscr{F}$-RCE.

For finite arrays we define the shift operator $\Gamma_{i j}$ by

$$
\Gamma_{i j} \circ X=\left\{X_{u v} ; i<u \leq m \text { or } j<v \leq r\right\} .
$$

Further define the reflection operator $R_{i j}$, where $R_{i j} \circ X$ produces the shell $\left\{X_{u v}^{\prime}\right.$; $i<u \leq m$ or $j<v \leq r\}$ where

$$
X_{u v}^{\prime}= \begin{cases}X_{m+i+1-u, r+j+1-v}, & u>i, v>j, \\ X_{u, r+j+1-v}, & u \leq i, v>j, \\ X_{m+i+1-u, v}, & u>i, v \leq j .\end{cases}
$$

DEFINITION. The $m \times r$ array $X$ has the strong reflection property if for any adapted random time $(S, T), \Gamma_{S, T} \circ X \stackrel{\mathscr{D}}{=} R_{S, T} \circ X,(0 \leq S \leq m-1,0 \leq T \leq r-1)$.

THEOREM 2. Let $X$ be a finite array. $X$ is $\mathscr{F}$-RCE if and only if $X$ has the strong reflection property.

PROOF. Let $X$ be $\mathscr{F}$-RCE and let $(S, T)$ be an adapted random time. Let $G_{i j}$ denote the space of the shells $\left\{X_{u v} ; i<u \leq m\right.$ or $\left.j<v \leq r\right\}$. Let $f: \bigcup_{i=0}^{m-1} \bigcup_{j=0}^{r-1} G_{i j} \rightarrow R$ be bounded. Then

$$
\begin{aligned}
E f\left(\Gamma_{S, T} \circ X\right) & =\sum_{i=0}^{m-1} \sum_{j=0}^{r-1} E\left[f\left(\Gamma_{i j} \circ X\right) ;(S, T)=(i, j)\right] \\
& =\sum_{i=0}^{m-1} \sum_{j=0}^{r-1} E\left[E\left(f\left(\Gamma_{i j} \circ X\right) \mid \mathscr{F}_{i j}\right) ;(S, T)=(i, j)\right] \\
& =\sum_{i=0}^{m-1} \sum_{j=0}^{r-1} E\left[E\left(f\left(R_{i j} \circ X\right) \mid \mathscr{F}_{i j}\right) ;(S, T)=(i, j)\right] \\
& =E f\left(R_{S, T} \circ X\right) .
\end{aligned}
$$

Thus $X$ satisfies $\Gamma_{S, T} \circ X \stackrel{\mathscr{D}}{=} R_{S, T} \circ X$.

Conversely, suppose $X$ has the strong reflection property. Let $A \in \mathscr{F}_{i j}$ and set $(S, T)=(i, j) I(A)+(m-1, r-1) I\left(A^{c}\right)$, for some $i \leq m-1, j \leq r-1$.

$$
\begin{aligned}
E f\left(\Gamma_{S, T} \circ X\right) & =E\left(f\left(\Gamma_{i j} \circ X\right) I(A)\right)+\left(E\left(f\left(\Gamma_{m-1, r-1} \circ X\right) I\left(A^{c}\right)\right)\right. \\
& =E\left(f\left(R_{i j} \circ X\right) I(A)\right)+\left(E\left(f\left(R_{m-1, r-1} \circ X\right) I\left(A^{c}\right)\right),\right.
\end{aligned}
$$


as $\Gamma_{S, T} \circ X \stackrel{\mathscr{Q}}{=} R_{S, T} \circ X$. Thus

$$
E f\left(\Gamma_{i j} \circ X\right) I(A)=E f\left(R_{i j} \circ X\right) I(A) \quad \text { for } A \in \mathscr{F}_{i j},
$$

so $\left(E\left(f\left(\Gamma_{i j} \circ X\right) \mid \mathscr{F}_{i j}\right)=E\left(f\left(R_{i j} \circ X\right) \mid \mathscr{F}_{i j}\right)\right.$.

Let $\pi_{k}$ denote the permutation mapping $(1,2, \ldots, m)$ to $(1,2, \ldots, k, m, m-1$, $\ldots, k+1)$ and let $\sigma_{\ell}$ denote the permutation mapping $(1,2, \ldots, r)$ to $(1,2, \ldots$, $\ell, r, r-1, \ldots, \ell+1)$. For fixed $(u, v) \leq(m, r)$ and $B_{i j} \in \mathscr{S}, i>u$ or $j>v$, and any $k \geq u, \ell \geq v$ consider

$$
\begin{aligned}
& P\left(\bigcap_{(i, j) \in A_{u v}}\left\{X_{i j} \in B_{i j}\right\} \mid \mathscr{F}_{u v}\right), \text { where } A_{u v}=\{(i, j): u<i \leq m \text { or } v<j \leq r\} . \\
& P\left(\bigcap_{(i, j) \in A_{u v}}\left\{X_{i j} \in B_{i j}\right\} \mid \mathscr{F}_{u v}\right)=E\left(\prod_{u<i \leq k} \prod_{v<j \leq \ell} I\left\{X_{i j} \in B_{i j}\right\} .\right. \\
&\left.E\left[\prod_{(i, j) \in A_{k \ell}} I\left\{X_{i j} \in B_{i j}\right\} \mid \mathscr{F}_{k \ell}\right] \mid \mathscr{F}_{u v}\right) \\
&=E\left(\prod_{u<i \leq k} \prod_{v<j \leq \ell} I\left\{X_{i j} \in B_{i j}\right\} .\right. \\
&=E\left(\prod_{(i, j) \in A_{k \ell}} I\left\{X_{i j} \in B_{\left.\pi_{k}(i) \sigma_{\ell}(j)\right\}} \mid \mathscr{F}_{k \ell}\right] \mid \mathscr{F}_{u v}\right) \\
&\left.=E\left(X_{i j} \in B_{\pi_{k}(i) \sigma_{\ell}(j)}\right\} \mid \mathscr{F}_{u v}\right) .
\end{aligned}
$$

Any pair of permutations $(\pi, \sigma)$ leaving $(1, \ldots, u)$ and $(1, \ldots, v)$ fixed, respectively, can be obtained by compositions of $\pi_{k}^{\prime} s$ and $\sigma_{\ell}^{\prime} s$ for $k>u, \ell>v$. Thus it follows that $\Gamma_{u v} \circ X$ is finitely, conditionally RCE given $\mathscr{F}_{u v}$.

DEFINITION. The $n \times n$ array $X$ has the weak reflection property if, for any $\left\{\mathscr{F}_{i, i}\right\}$ stopping time $S, 0 \leq S \leq n-1, \Gamma_{S, S} \circ X \stackrel{\mathscr{D}}{=} R_{S, S} \circ X$.

THEOREM 3. Let $X$ be an $n \times n$ array. $X$ is $\mathscr{F}$-weakly exchangeable if and only if $X$ satisfies the weak reflection property.

PROOF. The proof proceeds precisely as the proof of Theorem 2 above. Let $S$ be an $\left\{\mathscr{F}_{i i}\right\}$ stopping time and assume $X$ is $\mathscr{F}$-weakly exchangeable. Let $f: \bigcup_{i=0}^{n-1} G_{i i} \rightarrow R$ 
be a bounded function. Then

$$
\begin{aligned}
E f\left(\Gamma_{S, S} \circ X\right) & =\sum_{i=1}^{n-1} E\left[f\left(\Gamma_{i i} \circ X\right) ; S=i\right] \\
& \left.=\sum_{i=1}^{n-1} E\left[f\left(R_{i i} \circ X\right) \mid \mathscr{F}_{i i}\right) ; S=i\right] \\
& =E\left[f\left(R_{S, S} \circ X\right)\right],
\end{aligned}
$$

so $\Gamma_{S S} \circ X \stackrel{\mathscr{D}}{=} R_{S S} \circ X$.

Next suppose $X$ has the weak reflection property. Let $A \in \mathscr{F}_{i i}$ and set $S=$ i $I(A)+(n-1) I\left(A^{c}\right)$. As before we deduce

$$
E f\left(\Gamma_{i i} \circ X\right) I(A)=E f\left(R_{i i} \circ X\right) I(A)
$$

and so $E\left(f\left(\Gamma_{i i} \circ X\right) \mid \mathscr{F}_{i i}\right)=E\left(f\left(R_{i i} \circ X\right) \mid \mathscr{F}_{i i}\right)$.

For fixed $u \leq n, B_{i j} \in \mathscr{S}, i>u$ or $j>u$, and any $k \geq u$, if $\pi_{k}$ denotes the permutation mapping $(1,2, \ldots, n)$ to $(1,2, \ldots, k, n, n-1, \ldots, k+1)$,

$$
\begin{aligned}
& P\left(\bigcap_{(i, j) \in A_{u u}}\left\{X_{i j} \in B_{i j}\right\} \mid \mathscr{F}_{u u}\right)= E\left(\prod_{\substack{u<i<k, u<j \leq k}} I\left\{X_{i j} \in B_{i j}\right\}\right. \\
&\left.E\left[\prod_{(i, j) \in A_{k k}} I\left\{X_{i j} \in B_{i j}\right\} \mid \mathscr{F}_{k k}\right] \mid \mathscr{F}_{u u}\right) \\
&=E\left(\prod_{(i, j) \in A_{u u}} I\left\{X_{i j} \in B_{\pi_{k}(i), \pi_{k}(j)}\right\} \mid \mathscr{F}_{u u}\right)
\end{aligned}
$$

and so we can conclude $\Gamma_{i i} \circ X$ is conditionally weakly exchangeable given $\mathscr{F}_{i i}$.

As in the infinite case, we have the following result.

LEMMA 3. For a finite array $X$ adapted to a filtration $\mathscr{F}$ satisfying (F4), if the columns of $X$ are $\mathscr{F}^{1}$-exchangeable and the rows are $\mathscr{F}^{2}$-exchangeable, then $X$ is $\mathscr{F}$-RCE.

Proof. Given $(i, j)$, let $\pi$ be a permutation of $(1,2, \ldots, m)$ that leaves $(1, \ldots, i)$ fixed and let $\sigma$ be a permutation of $(1,2, \ldots, r)$ that leaves $(1, \ldots, j)$ fixed. Recall 
$A_{i j}=\{(u, v): i<u \leq m$ or $j<v \leq r\}$. For sets $B_{u v} \in \mathscr{S}$ and $(u, v) \in A_{i j}$,

$$
\begin{aligned}
& E\left(\prod_{(u, v) \in A_{i j}} I\left\{X_{u v} \in B_{u v}\right\} \mid \mathscr{F}_{i j}\right)= E\left(E\left(\prod_{(u, v) \in A_{i j}} I\left\{X_{u v} \in B_{u v}\right\} \mid \mathscr{F}_{i}^{1}\right) \mid \mathscr{F}_{j}^{2}\right) \\
&= E\left(\prod_{u \leq i, v>j} I\left\{X_{u v} \in B_{u v}\right\} .\right. \\
&\left.E\left(\prod_{u>i} \prod_{j} I\left\{X_{u v} \in B_{u v}\right\} \mid \mathscr{F}_{i}^{1}\right) \mid \mathscr{F}_{j}^{2}\right) \\
&= E\left(\prod_{u>i, v \leq j} I\left\{X_{\pi(u), v} \in B_{u v}\right\} .\right. \\
&\left.E\left(\prod_{i} \prod_{v>j} I\left\{X_{\pi(u), v} \in B_{u v}\right\} \mid \mathscr{F}_{j}^{2}\right) \mid \mathscr{F}_{i}^{1}\right) \\
&=E\left(\prod_{(u, v) \in A_{i j}} I\left\{X_{\pi(u), \sigma(v)} \in B_{u v}\right\} \mid \mathscr{F}_{i j}\right) .
\end{aligned}
$$

For general $\mathscr{F}$ the converse does not hold. However if $X$ is an $\mathscr{F}$-RCE array generated by random, independent, uniform permutations of the rows and columns of an array of fixed constants, that is, $X$ is an ergodic array, and $\mathscr{F}_{i j}=\sigma\{\pi(1), \ldots, \pi(i)$; $\sigma(1), \ldots, \sigma(j)\}$ then the columns of $X$ are $\mathscr{F}^{1}$-exchangeable and the rows are $\mathscr{F}^{2}$ exchangeable.

We continue to consider finite ergodic arrays. Let $\mathscr{F}$ be a filtration such that $\sigma\{\sigma(1), \ldots, \sigma(j)\} \subseteq \mathscr{F}_{0 j}$ and $\sigma\{\pi(1), \ldots, \pi(i)\} \subseteq \mathscr{F}_{i 0}$, for $i=1, \ldots, m ; j=$ $1, \ldots, r$. For this structure Theorem 4 provides a simple characterization of weak $\mathscr{F}$-RCE exchangeability analogous to Proposition 2.3 in Kallenberg [2].

DEFINITION. The random time $(S, T)$ is $\mathscr{F}$-predictable if $\{S=i, T=j\} \in$ $\mathscr{F}_{i-1, j-1}, i \geq 1, j \geq 1$.

THEOREM 4. Given $X$ as above, $X$ is weak $\mathscr{F}-\mathrm{RCE}$ if and only if $X_{S, T} \stackrel{\mathscr{Q}}{=} X_{11}$, for every $\mathscr{F}$-predictable random time $(S, T)$.

PROOF. Assume $X$ is $\mathscr{F}$-weak RCE. Then for any bounded function $f: G \rightarrow R$,

$$
\begin{aligned}
E f\left(X_{S, T}\right) & =\sum_{i} \sum_{j} E\left(f\left(X_{i j}\right) ;(S, T)=(i, j)\right) \\
& =\sum_{i} \sum_{j} E\left(E\left(f\left(X_{i j}\right) \mid \mathscr{F}_{i-1, j-1}\right) ;(S, T)=(i, j)\right)
\end{aligned}
$$




$$
\begin{aligned}
& =\sum_{i} \sum_{j} E\left(E\left(f\left(X_{m, r}\right) \mid \mathscr{F}_{i-1, j-1}\right) ;(S, T)=(i, j)\right) \\
& =E f\left(X_{m, r}\right) \\
& =E f\left(X_{11}\right)
\end{aligned}
$$

and so $X_{S, T} \stackrel{\mathscr{D}}{=} X_{11}$.

Conversely, assume $X_{S, T} \stackrel{\mathscr{D}}{=} X_{11}$ for all $\mathscr{F}$-predictable times $(S, T)$. From the proof of Theorem 1 , if

$$
M_{u v}=E\left[f\left(X_{u+1, v+1}\right) \mid \mathscr{F}_{u, v}\right], \quad 0 \leq u \leq m-1, \quad 0 \leq v \leq r-1,
$$

then $(M, \mathscr{F})$ is a martingale and

$$
E\left(f\left(X_{u v}\right) \mid \mathscr{F}_{i j}\right)=M_{i j}, \quad \text { for all } 0 \leq i<u \leq m, 0 \leq j<v \leq r .
$$

If we condition on $\mathscr{F}_{i j}$, then the first $i$ rows and $j$ columns are known, so the values of $X$ in the set $\left\{X_{u v}: i<u \leq m\right.$ and $\left.j<v \leq r\right\}$ are known. Thus, if we can show (8) implies $X$ is RCE, then a similar argument shows $X$ is weak $\mathscr{F}$-RCE.

Let $p$ and $q$ be fixed permutations of $(1, \ldots, m)$ and $(1, \ldots, r)$, respectively. We need to show $P(\pi=p, \sigma=q)=1 /(m ! r !)$.

First note $(\pi(m)=p(m), \sigma(r)=q(r))$ is $\mathscr{F}_{m-1, r-1}$ measurable and

$$
P(\pi(m)=p(m), \sigma(r)=q(r) \mid \pi(i)=p(i), \sigma(j)=q(j) ; i \leq m-1, j \leq r-1)=1 .
$$

Now

$$
\begin{aligned}
P(\pi(m-1) & =p(m-1), \sigma(r)=q(r) \mid \pi(i)=p(i), \sigma(j)=q(j) ; i \leq m-2, j \leq r-1) \\
& =1 / 2 .
\end{aligned}
$$

This can be seen by applying (8) with $f\left(X_{u v}\right)=I\left(X_{u v}=a_{i j}\right)$, where $a_{i j}$ is a value in the array $X$, since $E\left(f\left(X_{m r}\right) \mid \mathscr{F}_{m-2, r-1}\right)=E\left(f\left(X_{m-1, r}\right) \mid \mathscr{F}_{m-2, r-1}\right)$. Continuing in this way, $P(\pi=p, \sigma(r)=q(r) \mid \sigma(j), j \leq r-1)=1 / m !$.

Now $P(\sigma(r-1)=q(r-1) \mid \sigma(j), j \leq r-2)=1 / 2$, from $(8)$, as $E\left(f\left(X_{1 r}\right) \mid \mathscr{F}_{0, r-2}\right)$ $=E\left(f\left(X_{1, r-1}\right) \mid \mathscr{F}_{0, r-2}\right)$. So working down the first column of the array we find $P(\sigma=q, \pi=p)=(r ! m !)^{-1}$, as required.

If $X$ is a square $m \times m$ array then $\sigma=\pi$ a.s. if the diagonal set $\left\{X_{11}, X_{22}, \ldots, X_{m m}\right\}$ is deterministic. Under such a condition the following result gives a condition which ensures that the permutation is uniform on $(1,2, \ldots, m)$.

THEOREM 5. Suppose $\left\{X_{11}, \ldots, X_{m m}\right\}$ is deterministic. Then $X$ is weak $\mathscr{F}$-weakly exchangeable if and only if $X_{S, S} \stackrel{\mathscr{P}}{=} X_{11}$ for every stopping time $S$ predictable with respect to $\left\{\mathscr{F}_{i i}\right\}$. 
PROOF. The permutation $\pi$ is determined in this case by the diagonal. Thus the result follows by applying Proposition 2.3 of Kallenberg [2] to the diagonal.

\section{Continuous parameter processes}

Exchangeability may be defined for a 2-parameter process $\left\{X_{s, t}: s, t \in K\right\}$, where $K$ is $R_{+}^{2}$ or $[0,1]^{2}$. For any function $f(s, t)$ and any rectangle $B=\left(s_{1}, s_{2}\right] \times\left(t_{1}, t_{2}\right]$, let $f(B)$ denote the increment of $f$ over $B$ :

$$
f(B)=f\left(s_{2}, t_{2}\right)-f\left(s_{1}, t_{2}\right)-f\left(s_{2}, t_{1}\right)+f\left(s_{1}, t_{1}\right) .
$$

For fixed $\delta>0$, let $B_{i, j}=((i-1) \delta, i \delta] \times((j-1) \delta, j \delta]$. We shall say that the process $X$ is separately exchangeable if the array of increments of $X$ is RCE with respect to any square grid $\left(B_{i, j}\right)$, and $X$ is jointly exchangeable if the array of increments is weakly exchangeable.

In order to generalize the results of Sections 2 and 3, we shall assume henceforth that $X$ is 'continuous from above with limits from below' adapted to a filtration $\mathscr{F}=\left\{\mathscr{F}_{s, t}\right\}$, which is continuous from above. By this, we mean that

$$
\mathscr{F}_{s, t}=\bigcap_{u>s, v>t} \mathscr{F}_{u, v}, X_{s+, t+}, X_{s-, t+}, X_{s+, t-}, X_{s-, t-}
$$

all exist, and $X_{s+, t+}=X_{s, t}, \forall(s, t)$. It is also assumed that $X$ is 0 on the axes (that is, $X_{s, 0}=X_{0, t}=0, \forall s, t$ ), and if $X$ is defined on $[0,1]^{2}$, that $X$ is 'continuous from below' on the upper and right boundaries of the square (that is, $X_{s, 1}=X_{s-, 1-}=X_{s+, 1-}$ and $X_{1, t}=X_{1-, t-}=X_{1-, t+}$, for all $0<s, t<1$, and $\left.X_{1,1}=X_{1-, 1-}\right)$. We may define (weak) $\mathscr{F}$-exchangeability in the natural way: we say that $X$ is (weak) $\mathscr{F}$-separately (jointly) exchangeable if the corresponding array of increments over any square grid is (weak) $\mathscr{F}$-RCE (weakly exchangeable).

We begin with the continuous-parameter version of Theorem 1 for processes on $R_{+}^{2}$. Define $\Theta_{s, t}$ by $\left(\Theta_{s, t} \circ X\right)_{u, v}=X_{s+u, t+v}-X_{s, t+v}-X_{s+u, t}+X_{s, t}$. A random vector $(S, T)$ taking values in $R_{+}^{2}$ is an adapted random time with respect to $\mathscr{F}$ if $\{S \leq s, T \leq t\} \in \mathscr{F}_{s, t} \forall s, t \geq 0$. (Note: This definition is equivalent to the previous one in the case that the random time takes on at most countably many values.) It is easily shown that arbitrary adapted random times may be approximated from above by adapted random times taking on countably many values.

THEOREM 6. Let $X$ be a process defined on $R_{+}^{2} . X$ is weak $\mathscr{F}$-separately exchangeable if and only if $\Theta_{S, T} \circ X \stackrel{\mathscr{P}}{=} X$ for all adapted bounded random times $(S, T)$. 
Proof. Firstly assume that $X$ is weak $\mathscr{F}$-separately exchangeable and let $(S, T)$ be a bounded adapted random time. For adapted random times taking on dyadic values of the form $\left\{\left(i 2^{-n}, j 2^{-n}\right), i, j \leq m\right\}$, we may apply Theorem 1. For general stopping times, we approximate from above and use the fact that $X$ is continuous from above.

The converse is an easy corollary of Theorem 1 .

It is easily seen that there is a continuous-parameter version of Lemma 2.

We now consider processes defined on $[0,1]^{2}$. The shift operator $\Gamma_{s t}$ is defined as follows: $\Gamma_{s t} \circ X=\left\{X_{u v}^{\prime}, u \geq s\right.$ or $\left.v \geq t\right\}$, where $X_{u v}^{\prime}=X_{u v}-X_{u \wedge s, v \wedge t}$, if $u \geq s$ or $v \geq t$. Note $X_{u v}^{\prime}$ denotes the increment over the possibly $L$-shaped region that we need to consider for processes defined on $[0,1]^{2}$.

The reflection operator $R_{s t}$ is defined as follows: $R_{s t} \circ X=\left\{X_{u v}^{\prime \prime}, u \geq s\right.$ or $\left.v \geq t\right\}$, where

$$
X_{u v}^{\prime \prime}= \begin{cases}X_{1,1}-X_{(1+s-u)-,(1+t-v)-}, & u \geq s, v \geq t, \\ X_{1, v}-X_{(1+s-u)-, v}, & u \geq s, v<t, \\ X_{u, 1}-X_{u,(1+t-v)-}, & v \geq t, u<s .\end{cases}
$$

$X$ is said to have the strong reflection property if $\Gamma_{S T} \circ X \stackrel{\mathscr{Q}}{=} R_{S T} \circ X$ for all adapted random times $(S, T) . X$ is said to have the weak reflection property if $\Gamma_{S S} \circ X \stackrel{\mathscr{D}}{=} R_{S S} \circ X$ for all (1-dimensional) stopping times with respect to the filtration $\left\{\mathscr{F}_{s, s}, 0 \leq s \leq 1\right\}$.

The analogue of Theorem 2 is:

THEOREM 7. Let $X$ be a process defined on $[0,1]^{2} . X$ is $\mathscr{F}$-separately exchangeable if and only if $X$ has the strong reflection property.

ProOF. Assume first that $X$ has the strong reflection property. Apply Theorem 2 to the increments over any square grid, using discrete stopping times.

Now assume that $X$ is $\mathscr{F}$-separately exchangeable. Apply Theorem 2 to increments of the form $X_{1-(k-1) h, 1-(j-1) h}-X_{1-k h, 1-(j-1) h}-X_{1-(k-1) h .1-j h}+X_{1-k h .1-j h}$, where $X$ is continuous a.s. at $(1-k h, 1-j h), k, j \geq 1$. Noting that the set of such $h$ is dense in $[0,1]$, we may approximate arbitrary random times from above to obtain the same conditional finite dimensional distributions for $\Gamma_{S T} \circ X$ and $R_{S T} \circ X$.

The analogue of Theorem 3 is shown in exactly the same way:

THEOREM 8. Let $X$ be a process defined on $[0,1]^{2} . X$ is $\mathscr{F}$-jointly exchangeable if and only if $X$ has the weak reflection property. 


\section{References}

[1] D. Dacunha-Castelle, 'Indiscernability and exchangeability in $L^{p}$ spaces', in: Proc. Seminar on Random Series, Convex Sets and Geometry of Banach Spaces (Aarhus, 1974) pp. 50-56.

[2] O. Kallenberg, 'Characterizations and embedding properties in exchangeability', Z. Wahrsch. Verw. Gebiete 60 (1982), 249-281.

[3] J. F. C. Kingman, 'Uses of exchangeability', Ann. Probab. 6 (1978), 183-197.

Department of Mathematics

University of Ottawa

Ottawa, Ontario

Canada K1N 6N5
School of Mathematics and Statistics

University of Sydney

NSW 2006

Australia 